\title{
Quorum based Distributed Mutual Exclusion Algorithms in Mobile Networks
}

\author{
Rachita Juneja \\ Scientist, SAG, DRDO \\ Metcalfe House, Delhi-54
}

\author{
Vinod Kumar \\ Associate Professor, \\ Computer Engineering Department, Delhi \\ Technological University, Delhi
}

\begin{abstract}
The assigned frequency spectrum to the wireless mobile systems has become a scarce resource as the number of mobile users has increased tremendously. So there is a need of using the allotted bandwidth efficiently. Distributed RME assigns channels to various cells and increases bandwidth utilization and at the same time reduces co- channel interference. Various algorithms exist which help in attaining mutual exclusion. Quorum based algorithms is one such class of algorithms where the requesting site asks permission from a set of smaller number of participating sites called a quorum. Quorums help reduce the message complexity in mobile systems.
\end{abstract}

\section{Keywords}

Co-channel interference, Minimum reuse distance, Euclidean distance, Relaxed Mutual Exclusion, Dynamic channel allocation, Quorum, Coterie, Timestamps.

\section{INTRODUCTION}

The assigned frequency spectrum to the wireless mobile systems has become a scarce resource as the number of mobile users has increased tremendously. So there is a need of using the allotted bandwidth efficiently. A general idea about geographical division of cellular communication network consists of clusters of hexagonal cells each having a fixed base station called mobile service station (MSS)[2],[5],[7]. All the MSS's are connected to each other through a fixed communication network. When a mobile host $(\mathrm{MH})$ wants to establish a call, it sends a request to MSS. If a free channel is available with the MSS, it grants the channel to the MH which then proceeds with the call. If a particular channel is being used by more than one $\mathrm{MH}$ at the same time in a cell or the neighbouring cells, the calls will interfere with each other. Such interference is called co-channel interference [1], [8]. The use of particular frequency in a cell for communication session establishment can be viewed as equivalent to entering the critical section by the cell in which the channel is being used. In mobile communication, frequency channels are the common resource. Each cell has to attain a frequency channel for communication. Since two or more neighbouring cells can try to attain the same channel at the same time, this can be viewed as similar to Mutual Exclusion Problem where the processes wait for a shared resource currently being used by some other process in order to complete their task.

The main challenge, the mobile technology is facing today is the effective utilization of bandwidth as the number of mobile users are increasing at an electrifying speed. To enable large number of users to communicate efficiently without call blocks/drops, frequencies can be reused between cells separated by a minimum reuse distance $\mathrm{D}_{\min }$. An $\mathrm{x}^{*} \mathrm{y}$ cellular network has $\mathrm{x}$ rows and $\mathrm{y}$ columns of cells. Cell at $\mathrm{i}^{\text {th }}$ row and $\mathrm{j}^{\text {th }}$ column is denoted as $(\mathrm{i}, \mathrm{j})$. The distance between the cells is defined as the Euclidean distance between the centres of two cells [2]. The distance between any two cells $\mathrm{C}_{1}=\left(\mathrm{i}_{1}, \mathrm{j}_{1}\right)$ and $\mathrm{C}_{2}=\left(\mathrm{i}_{2}, \mathrm{j}_{2}\right)$ is

Dist $\left(C_{1}, C_{2}\right)=\sqrt{ }\left(i_{1}-i_{2}\right)^{2}+\left(i_{1}-i_{2}\right)\left(j_{1}-j_{2}\right)+\left(j_{1}-j_{2}\right)^{2}($ eqn. 1)

Let the centres of two cells be $(0,0)$ and $(a, b)$. Then distance between them

$D_{\min }=\sqrt{ }\left(a^{2}+a * b+b^{2}\right)$ using eqn. 1

This distance is called the minimum reuse distance [2]. The nearest co-channel cells to a cell are those cells whose separating distance is exactly $\mathrm{D}_{\text {min }}$. The distance between two cells is defined as the distance between the centres of the cells. A channel can be reused in any two cells if the distance between them is at least $D_{\min }$. This means that two channels having a separation of greater than or equal to $D_{\min }$ can use the same frequency channel at the same time without any interference in order to achieve good quality communication between the mobile users. This enhances the use of allotted bandwidth. Two cells having a separating distance $<D_{\min }$ cannot use the same frequency as their calls would interfere with each other. So adjacent cells are not allotted same frequencies.

Frequency reuse leads to the idea of relaxed mutual exclusion (RME) since multiple distinct critical sections can be executed concurrently in the cells separated by distance greater than or equal to $\mathrm{D}_{\min }$. In mobile communications, RME is used instead of Mutual Exclusion (ME) since some cells can use particular channel concurrently while others are not allowed to do so. Thus ME can be considered as a special case of RME. In ME two processes cannot use the same resource at the same time.

The bandwidth assignment in a mobile cellular system can be static, dynamic or the hybrid of the two. Static channel allocation means fixed channels are permanently assigned to each cell in order to handle the calls. When a MH requests for a call establishment, free channels in the allotted band are searched. If such a channel is available, call can be established. If no free channel is available in the cell, the call is dropped. Such a scheme cannot handle the increasing traffic load. So dynamic channel allocation is used where channels can move between the cells according to the traffic load in the cells. As cells can use each other's free channels, each cell must maintain a list of its own available channels and also that of its neighbours. In order to maintain such huge database for implementing dynamic channel allocation, either centralized or distributed dynamic channel allocation schemes are used. In centralized dynamic channel allocation, the mobile switching centre (MSC) is the centralized authority which 
contains all information about available channels in the mobile system. It assigns the channels from this pool and later on when the channels are released after usage, they are returned back to this common pool of available channels. As centralized scheme suffers from a single point of failure at the MSC, distributed dynamic channel allocation (DDCA) schemes are used. In DDCA each cell maintains lists of available and busy channels of itself and its neighbours. When a call needs to be established, a channel is searched from these lists. If a free channel is available, it is assigned to that call. This scheme is called DDCA since message passing is used to exchange the status of channels at any instant among various cells. In hybrid scheme, some channels in a cell are permanently allotted while others are dynamically assigned.

\section{DME ALGORITHMS}

DDCA can be considered as an application of RME. Here a cell wants to be assigned a channel for call establishment. It does not matter from where the channel is obtained as long as there is no interference between various calls.

In distributed RME (DRME), the critical resources need to be assigned to different sites such that at a particular site, two or more processes cannot use the same critical resource at the same time. Also such a resource can be used among various different sites at the same time as long as they are noninterfering. So distributed RME assigns channels to various cells and increases bandwidth utilization and at the same time reduces channel interference.

Since RME is a generalization of ME, a DME algorithm can be generalized to design a DRME algorithm.

There are two classes of DME algorithms:

1. Token based

2. Permission (non-token) based

The permission based DME algorithms are further classified as voting based and coterie based algorithms

Token based algorithms achieve ME using a privilege message called token which is shared among all the participating sites. Since there is a single token for the entire system ME is guaranteed. No two sites can possess the token at the same time. The token based algorithms do not find effective use in channel allocation as frequency reuse is being done in order to maximise bandwidth utilization. A single token should exist in a system is the basis of token based algorithms which is violated in channel allocation (as frequency reuse is applicable here).

Permission based algorithms need permission from participating sites in order to execute critical section (CS). When a process in a site wants to enter CS, it has to acquire permission from all the participating sites. If all these sites agree, only then can a process enter CS. When some of the participating sites themselves want to enter CS, timestamps are used to avoid conflict. If the timestamp of requesting site is higher than any of the participating sites which also want to execute $\mathrm{CS}$, it is not granted permission.

Permission based algorithms can further classified as voting based and coterie based algorithms. In voting based algorithms each participating site is assigned a non- negative integer called a vote. Permission needs to be taken from the participating sites such that the sum total of votes acquired by a requesting site is simple majority to the total number of votes in the system. Thus the voting algorithms use majority voting to achieve ME. A site asking for permission to execute CS does not worry as to which sites vote for it. What it really worries is that it should get majority votes in the system. Such a scheme of majority voting given by Thomas [13] assigns uniform votes (i.e. equal weight age) to the entire system. Another scheme given by Gifford uses weighted voting in which some of the participating sites in the system are assigned different votes than other sites [12]. This technique is called quorum consensus method.

In coterie based permission algorithms, a sub-group of sites is constituted according to some rule and the requesting site needs to acquire grant messages from this sub group only rather than the entire system. Such a sub- group is called a quorum. A set of quorums constitutes a coterie. For example, $\{\{1,3\},\{1,4\},\{2,3\},\{2,4\}\}$ is a set of four quorums and is called a 2 -coterie [11]. In this coterie, $\{\{1,3\},\{2,4\}$ or $\{1$, $4\},\{2,3\}\}$ are two mutually disjoint quorums and thus a property called minimality property is satisfied. This property suggests that no quorum is a superset of another quorum in the coterie. This property helps in multiple entries to CS for mutually disjoint quorums. Thus quorums decrease the message complexity in the system which is limited to the size of the quorum. Different algorithms exist for constructing quorums.

Lamport gave an algorithm which is perhaps the first DME algorithm with message complexity of 3(N-1) [14]. If number of sites are $\mathrm{N}$, a site would send a request message to $\mathrm{N}-1$ sites which would (if willing) grant their messages to this site. Since N-1 sites received the request message and all these sites are willing to grant the resource, $\mathrm{N}-1$ grant messages would be received by this requesting site. Now the requesting site would send the release message to these $\mathrm{N}-1$ sites who granted it the permission to use the resource. So total number of messages communicated in the system are $3(\mathrm{~N}-1)$.

An algorithm given by Ricart and Agarwala is also an earliest known DME algorithm [9]. Here a requesting site is granted permission to enter the CS if the participating sites themselves do not want to execute $\mathrm{CS}$ at the time the requesting site sends request message. If any of the participating sites is interested in entering the CS, it checks the timestamp [10] of its requested message and the incoming request message. Lower time stamped site gets the chance to enter the CS. In this algorithm, the participating sites do not lock themselves exclusively after granting a particular request. They keep on granting permission to any number of sites till the resource is used. The message complexity of request- reply messages is $2(\mathrm{~N}-1)$ as $\mathrm{N}-1$ request messages are sent and $\mathrm{N}-1$ reply messages received by the requesting site. This algorithm is free of deadlocks and starvation but it is expensive in communication cost as the requesting sites communicate with all other sites in the system to enter the CS [11]. This algorithm by Ricart and Agarwala is based on 'self- conflict'. The participating site is worried only for the conflict between itself and the requesting site for entering the CS.

Since the participating sites do not grant exclusive locks to the requesting site, Maekawa proposed a DME algorithm where exclusive locks were granted for a requesting site and no other requests were entertained further. Only after obtaining RELEASE message from the requesting site would a participating site grant permission to enter CS to other requesting sites, based on the priority. Certain properties of 
constructing quorums must be satisfied so that $\mathrm{ME}$ is guaranteed [4]. The properties include

1. $\mathrm{Q}_{\mathrm{i}}$ is contained in $\mathrm{S}_{\mathrm{i}} \forall i \in 1,2,3 \ldots N$

2. $\mathrm{S}_{\mathrm{i}} \cap \mathrm{S}_{\mathrm{j}} \neq \emptyset \forall i, j \in 1,2, \ldots N$

3. $\left|\mathrm{S}_{\mathrm{i}}\right|=\mathrm{k} \forall i \in 1,2,3 \ldots N$ where $\mathrm{k}<\mathrm{N}$. This is called equal work property as each site will send and receive equal number of messages for achieving ME.

4. $\mathrm{Q}_{\mathrm{i}}$ is contained in $\mathrm{k} \mathrm{S}_{\mathrm{j}}$ 's $\forall i \in 1,2,3 \ldots N$. This is called equal responsibility property.

$\mathrm{N}$ is the number of sites, $\mathrm{Q}_{\mathrm{i}}$ refers to the $\mathrm{i}^{\text {th }}$ site of the communication network.

$\mathrm{S}_{\mathrm{i}}$ is a set of $\mathrm{k} \mathrm{Q}_{\mathrm{j}}$ 's $\forall i, j \in 1,2, \ldots N$

Maekawa explained that for a fixed $\mathrm{k}$, maximum possible value of $\mathrm{N}$ would be $\mathrm{k}(\mathrm{k}-1)+1$ with the assumption that any two quorums have only one intersection site. Hence the theoretical lower bound of quorum size is approximately $\sqrt{\mathrm{N}}$ [6]. Theoretically quorums can be generated by trying all combinations of the requesting sites which satisfy the above properties. Maekawa's original paper explains the construction of finite projective plane but not all projective planes exist [6]. So Maekawa gave another algorithm called grid based algorithm which avoids the construction of finite projective planes. Here the sites are organized as a grid of squares as shown in the figure. A quorum can be constructed by the union of row and column containing the requesting site. In this algorithm two sites $S_{i}$ and $S_{j}$ intersect each other in two sites $\forall i, j$. So any two quorums have two intersections. The quorum size is roughly twice the theoretical lower bound as proposed in the finite projective planes i.e. $2 \sqrt{\mathrm{N}}-1$. Though this algorithm is simple to understand, but any two quorums have two intersections here. So it is not properly optimized.

A better option has been suggested by Wai- Shing Luk and Tien- Tsin Wong is to construct a quorum using either a row or a column. Here $\mathrm{N}$ is no longer a perfect square. The sites can be organized in the form of a right angled triangle as shown in the figure. Starting from the leftmost node on the first row, move farthest right horizontally along the row and take a 90 degree turn (when no more nodes exist) to the bottom along the column. The line joining such a row and column contains the sites of a quorum. All such quorums can be formed starting at different rows. This scheme is called the row based scheme [4].

Another similar scheme is the column based triangle configuration [4]. Here a line is drawn starting from the rightmost and the bottommost node. This node takes a 90 degree turn to the left and covers the entire row (if there is no other node along its path in the column). Thus the line reaches the leftmost node staring from the farthest bottommost node. The nodes joined by this line constitute a quorum. Any two such lines meet at exactly one node. Therefore, any two quorums have exactly one intersection (property 2 ) in both the row and the column based schemes.

The size of the quorum is smaller near the top of the grid (only entire row included) in the row based scheme. Similarly, the size of the quorum is smaller at the bottom of the grid where only column of the sites is included. This causes a violation to the Property 4 which is the equal responsibility property. To solve this problem, a combination of row based and column based schemes is used. Here the requesting site does not have to tell other sites about whether it is using a row based scheme for quorum construction or the column based one. The quorum size is approximately $\sqrt{ } 2 \mathrm{~N}$ [4].

Another method to construct quorums assumes that the cellular system is organized as a binary tree. The quorum consists of a branch from the root node to the leaf node. This method is called the tree-quorum algorithm and has been given by Agrawala and Abbadi [15]. The size of the quorum is $\sqrt{ } \mathrm{N}$ here

A comparison of various permission based algorithms is given on the basis of performance metrics in table 1 .

Table 1. Comparison of various permission based algorithms

\begin{tabular}{|c|c|c|}
\hline Algorithm & $\begin{array}{c}\text { Message } \\
\text { complexity }\end{array}$ & $\begin{array}{c}\text { Synchronization } \\
\text { delay }\end{array}$ \\
\hline Lamport & $3(\mathrm{~N}-1)$ & 1 \\
\hline $\begin{array}{c}\text { Ricart and } \\
\text { Aggrawal }\end{array}$ & $2(\mathrm{~N}-1)$ & 1 \\
\hline Maekawa grid & $2 \sqrt{\mathrm{N}}$ & 2 \\
\hline $\begin{array}{c}\text { Luk Wong row- } \\
\text { column }\end{array}$ & $\sqrt{2 \mathrm{~N}}$ & 2 \\
\hline $\begin{array}{c}\text { Agrawala and } \\
\text { Abbadi Tree }\end{array}$ & $\sqrt{\mathrm{N}}$ & 2 \\
\hline
\end{tabular}

Where $\mathrm{N}$ is the number of sites in the system

\section{CONCLUSION}

Many different methods to construct the quorums have been discussed in this paper. The advantage of permission based mutual exclusion algorithms is that they exhibit excellent fault-tolerance and load-balancing characteristics. The main drawback of permission based mutual exclusion algorithms is that the communication cost to enter critical section is directly proportional to the size of quorums. It is hard to decide which algorithm is the best to achieve mutual exclusion and thus reduce the co-channel interference. Choice of a particular algorithm depends on the network topology, system requirements, performance measures viz., message complexity, communication delay, availability etc. The designer has to look at the implementation aspects also when zeroing down on a particular algorithm apart from the performance of the algorithm on various metrics. A performance metric, message complexity is dependent on the size of the quorum. The smaller the quorum size, the lesser is the message complexity. In the above mentioned quorum algorithms, Agrawala and Abbadi's tree based quorum algorithm has the lowest message complexity.

\section{REFERENCES}

[1] Ravi Prakash, Niranjan, G. Shivaratri, Mukesh Singhal, "Distributed Dynamic Channel Allocation for Mobile Computing”, 1995 ACM.

[2] Jianping Jiang, "On Distributed Dynamic Channel Allocation in Mobile Cellular Networks", IEEE transactions on Parallel And Distributed Systems, vol. 13, No. 10, 2002.

[3] P.C.Saxena, J.Rai, "A survey of permission- based distributed mutual exclusion algorithms", Computer Standards \& Interfaces 25 (2003) 159-181. 
[4] W. Luk, T. Wong, Two new quorum based algorithms for distributed mutual exclusion, Proceedings of the 17th International Conference on Distributed Computing Systems, ICDCS' 97, Baltimore, MD, USA, IEEE (1997, May),pp. 100-106.

[5] D.J Goodman, Wireless Personal Comm. Systems, Addison Wesley

[6] M. Maekawa," $A \sqrt{ } \mathrm{N}$ algorithm for mutual exclusion in decentralized systems", ACM Trans. Comput. Systs., pp 145-159, May 1985

[7] W.C.Y.Lee, Mobile Cellular Telecommunications: Analog and Digital Systems. McGraw - Hill

[8] V.H.MacDonald, "The Cellular Concept",The Bell System Technical J.., vol. 58, no. 1

[9] Ricart G and Agarwala, A. K. "An optimal algorithm for mutual exclusion in computer networks", CACM., vol. 24, no. 1,1981

[10] Kumar, A. " Hierarchical Quorum Consensus: a new algorithm for managing replicated data," IEEE Trans. Comp., vol. 40, no. 9, 1991

[11] Shing- Tsaan Huang, Jehu- Ruey Jiang and Yu- ChenKuo, National Tsing Hua university, Hsin Chu, Tiawaan , " k-coteries foe Fault- Tolerant k entries to a Critical Section”, NSC Republic of China.
[12] Gifford, D.K. "Weight voting for replicated data", in Proc. $7^{\text {th }}$ ACM SIGOPS Symp. Oper. Syst. Principles, Pacific Grove, CA, pp. 150- 159, 1979

[13] Thomas, R.H, "A majority consensus approach to concurrency control," ACM Trans. Database Syst., vol 4, no. 2, pp. 180-209,1979

[14] Lamport, L. "Time, clocks and the ordering of events in a distributed system, CACM., vol. 21, no. 7, pp. 145-159, July 1978

[15] Agrawala, D., and El Abbadi, "An efficient and faulttolerant algorithm for distributed mutual exclusion," ACM Trans. Comp. Syst., vol.9, no. 1, pp. 1-20, Feb.1991

[16] Handbook of Wireless Networks and Mobile Computing, Edited by Ivan Stojmenovic'Copyright (C) 2002 John Wiley \& Sons, Inc.

[17] H. Garcia-Molina, D. Barbara, "How to assign votes in a distributed system", Journal for the Association for Computing Machinery, 1985.

[18] Megna Gupta, A.K Sachan, Journal of Theoritical and Applied Information Technology, 2007, "Distributed Dynamic Channel Allocation Algorithm for Cellular Mobile Network".

[19] Jiangchang Yang et.al. "A fault tolerant channel allocation algorithm for cellular network with mobile base stations". IEEE transactions on vehicular technology 56(1): 349- 361, 2007. 